\title{
Mydriasert pupillary dilation for cataract surgery: an economic and clinical study
}

\author{
Ameet Shah ${ }^{1 \dagger}$, Sukhvinder Johal ${ }^{2^{* \dagger}}$ and Nicholas Lee ${ }^{3}$
}

\begin{abstract}
Background: Mydriasert is an insoluble ophthalmic insert indicated for mydriasis prior to cataract surgery, which gradually releases the active ingredients: tropicamide $(0.25 \mathrm{mg})$ and phenylephrine $(5.38 \mathrm{mg})$. This study aimed to evaluate the cost of Mydriasert compared with conventional mydriatic eye drops to induce pupil dilation prior to cataract surgery using a budget impact model.

Methods: A cohort-based, decision tree, budget impact model was developed to estimate the drug, consumable and staff costs for achieving mydriasis with Mydriasert compared to mydriatic eye drops (tropicamide [1 \%] plus phenylephrine [10\%]). Insights from structured interviews with clinicians $(n=5)$ experienced in using both Mydriasert and mydriatic eye drops and results from the current clinical study of patients undergoing cataract surgery $(n=144)$ at a Greater London district general hospital were used to obtain key input parameters for the model, and to validate the model approach.

Results: The base case analysis in a cohort of 1763 patients undergoing cataract surgery showed that when Mydriasert substituted mydriatic eye drops, annual total costs decreased by $18 \%$ and annual total nurse time decreased from 235.1 hours to 44.1 hours over one year (2012-2013).

Conclusions: This study demonstrated that despite its higher unit cost than mydriatic eye drops, Mydriasert resulted in overall savings in health-care costs, mainly associated with reduced nursing time. The economic model developed could assist National Health Service managers and local payers to estimate the budget impact of the introduction of Mydriasert into different clinical settings.
\end{abstract}

Keywords: Mydriasert, Mydriasis, Mydriatics, Mydriatic eye drops, Cataract surgery, Economic model

\section{Background}

Efficient and stable mydriasis is necessary during many diagnostic and surgical procedures in ophthalmology $[1,2]$. Mydriasert ${ }^{\circ}$ (Laboratoires Théa) is an insoluble ophthalmic insert indicated for pre-surgical mydriasis, which gradually releases the active ingredients: tropicamide $(0.25 \mathrm{mg})$ and phenylephrine $(5.38 \mathrm{mg})$ [3]. Published studies have demonstrated that Mydriasert is effective in achieving stable mydriasis compared to conventional topical mydriatic eye drop regimes [1-8] with a good safety profile [6] and a faster recovery of near vision after dilation of the pupil $[1,6]$.

\footnotetext{
* Correspondence: Sukhvinder.Johal@parexel.com

${ }^{\dagger}$ Equal contributors

${ }^{2}$ Health Economic Modelling Unit, HERON ${ }^{T M}$ Commercialization, PAREXEL International, London, UK

Full list of author information is available at the end of the article
}

The unit cost of Mydriasert is several times greater than that of eye drops [9]; however, there may be considerable cost-savings in nursing time for multiple instillations of pre-operative drops compared to a one-off insertion of a Mydriasert pellet.

This study aimed to evaluate the cost of Mydriasert compared to conventional mydriatic eye drops (tropicamide [1\%] plus phenylephrine [10 \%]) to induce pupil dilation prior to cataract surgery using a budget impact model. A prospective clinical evaluation of the introduction of Mydriasert for pre-operative pupillary dilation in a Greater London district general hospital ophthalmology department provided input parameters for the economic model. The economic model was designed to assist National Health Service (NHS) managers and local payers in considering the cost implications of introducing Mydriasert into different clinical settings. 


\section{Methods}

\section{Budget impact model structure}

A budget impact model was developed to estimate the drug, consumable and staff costs for achieving mydriasis with Mydriasert compared to mydriatic eye drops (tropicamide [1 \%] plus phenylephrine [10 \%]). The model was designed to give maximum flexibility to the user by allowing a choice of analysis perspectives (national, health commissioning board, local clinical commissioning groups or clinic etc.) and changes to all input parameters such as the proportion of patients treated with Mydriasert or mydriatic eye drops, drug prices and resource use/staff time.

The budget impact model was cohort-based, utilised a decision tree structure (Fig. 1) and followed patients from cataract surgery preparation to the post-surgery recovery period. It calculated costs for an estimated cohort of patients over a one year time horizon. The analysis aimed to capture all costs and outcomes relevant to the NHS to identify any differences in annual costs between the use of Mydriasert and mydriatic eye drops [10].

\section{Base case analysis}

In the base case analysis of a single London hospital population of 1763 patients undergoing cataract surgery, a direct comparison of costs of the two treatment options (treating all patients with Mydriasert compared to treating all patients with mydriatic eye drops $11 \%$ tropicamide and $10 \%$ phenylephrine]) was conducted. The main economic outcomes evaluated were total costs and nurse time saved.

\section{Budget impact model inputs}

Structured interviews with 5 clinicians experienced in using both Mydriasert and mydriatic eye drops based in different centres in the UK were conducted in February 2013. Insights from these interviews and results from a current clinical study [11] of patients $(n=144)$ undergoing cataract surgery (from September 2012 to April 2013) were used to obtain key input parameters for the model, and to validate the model approach. In addition, drug prices used in the model were obtained from the British National Formulary (2013) [9], and surgical consultant and nurse costs were obtained from the Personal Social Services Research Unit (2012) [12].

Input parameters included in the base case analysis are summarised in Table 1 . In the clinical study, a proforma for nursing staff (collected over 4 weeks), recorded the amount of time spent inserting the pellet and included a patient satisfaction score on a scale of 1 to 10 , with 1 indicating highly dissatisfied and 10 indicating highly satisfied. Surgical proformas recording pupil size according to the classification shown in Fig. 2a, time since Mydriasert insertion (for the first 50 cases) and any adverse events were registered by a single surgeon over 6 months [11]. Data was collected on all consecutive cases operated on over the 6 month period. Cases were deemed suitable for a senior specialty registrar and included eyes noted to dilate poorly in clinic and patients on agents known to cause intra-operative floppy iris syndrome such as tamsulosin and doxazocin. The study was carried out with the approval of the Research and Development committee at Hillingdon Hospital NHS Foundation Trust, London, UK.

\section{Budget impact model assumptions}

The underlying assumption for the budget impact model described was that Mydriasert and mydriatic eye drops had equivalent health and surgical outcomes for patients 
Table 1 Input parameters for the base case cost analysis of Mydriasert compared to eye drops for mydriasis during cataract surgery at a London hospital from 2012-2013

\begin{tabular}{ll}
\hline Inputs & Cost/Nalue \\
\hline Drug prices & \\
Mydriasert & $£ 4.20$ \\
Mydriatic drops (cost per vial) & \\
Tropicamide (1\%) & $£ 0.47$ \\
Phenylephrine (10\%) & $£ 0.49$ \\
Resource utilisation and costs & \\
Surgical consultant cost per minute & $£ 2.45$ \\
Nurse cost per minute & $£ 0.70$ \\
Mydriasert: number of instillations & 1 \\
Eye drops: number of instillations & 4 \\
Time to instil Mydriasert & 1.5 minutes \\
Time to instil eye drops & 2 minutes \\
Percentage of Mydriasert inserts that extrude & $2.6 \%$ \\
\hline
\end{tabular}

The majority of input parameters were obtained from the current clinical study or insights from clinician interviews with the exception of drug prices from the British National Formulary (2013)8 and surgical consultant and nurse costs obtained from the Personal Social Services Research Unit (2012) 10. ${ }^{a}$ For eye drops it was assumed one vial was used per patient

and therefore, adverse events, complications and mortality related to cataract surgery were not included in the model. It was also assumed that the time to remove the Mydriasert was negligible and added no extra time to surgery.

Parameters were varied in univariate sensitivity analyses to assess model robustness.

\section{Scenario analysis}

In the event that pupil dilation was not sufficient to perform cataract surgery, iris hooks were sometimes used to achieve a larger pupil. The additional costs of iris hook use were considered in a scenario analysis. Surgical theatre order books were reviewed to determine the number of iris hooks ordered in the 8 months preceding, and following the introduction of Mydriasert. The statistical significance of any change in iris hook orders (as a proportion of cataract operations performed) was determined using the chi-squared test.

\section{Results}

\section{Impact of mydriasert in clinical practice}

Ninety-seven nursing proformas were returned (mean patient age $=74.9$ years [range 49 years to 91 years]; $45 \%$ were female and $55 \%$ male) and 114 surgical proformas were completed (mean patient age $=72.6$ years [range 48 years to 91 years]; $41 \%$ were male, and $59 \%$ female) for the clinical evaluation of Mydriasert in cataract surgery.

The average nursing time spent inserting Mydriasert was $1.52 \pm 0.60$ minutes (mean $\pm \mathrm{SD}, \mathrm{n}=85$ ) which was
A

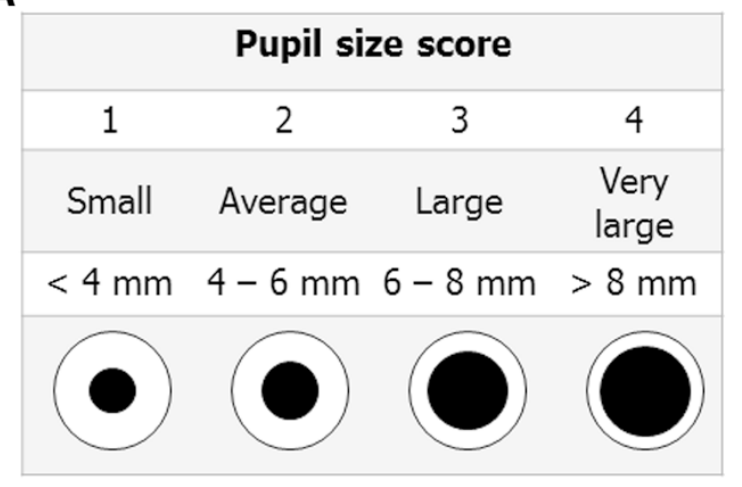

B

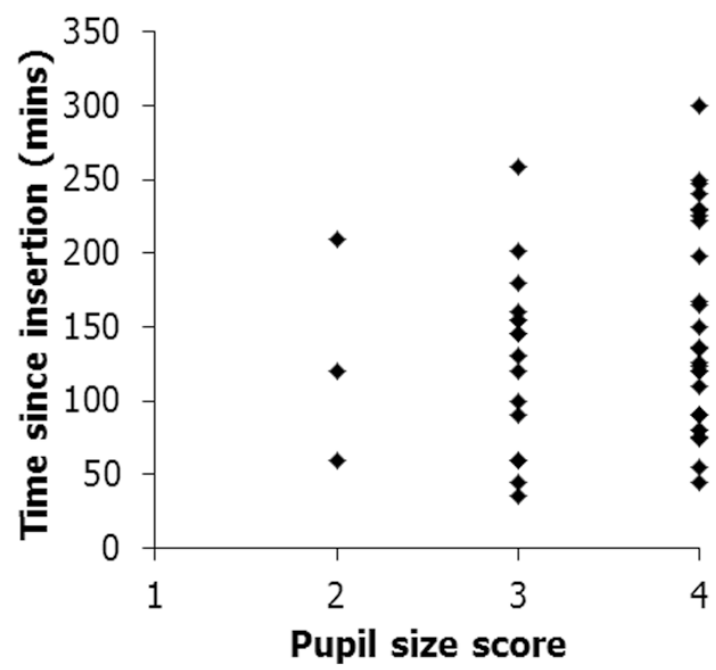

Fig. 2 Pupil size score a and scatter plot comparing pupil size score against time since Mydriasert insertion b. a Pupil size score was recorded at the start of surgery after Mydriasert insertion to evaluate the efficacy and record Mydriasert extrusions and $\mathbf{b}$ time since Mydriasert insertion was also recorded for the first 50 cases. Mins, minutes; mm, millimetre.

used as an input for the economic model (Table 1). Using Mydriasert to induce dilation, pupil size (defined by the pupil size score from 1 to 4 ) was generally large or very large (mean score $\pm S D, 3.32 \pm 0.66 ; n=114$ ) (Fig. 2a) which was sustained for up to 300 minutes after pellet insertion (Fig. 2b). Patients were very satisfied with the procedure based on a satisfaction score from 1 to 10 (mean score $\pm \mathrm{SD}, 8.97 \pm 1.32 ; \mathrm{n}=97$ ).

The insert was not found in three cases. In one case the anaesthetist forgot to the remove the insert, although it was subsequently discovered and removed at speculum placement. An area of epithelial irregularity was noted under the operating microscope at the start of one case and this had not been present when the patient had been examined at the slit lamp earlier that day. The patient was treated with a course of chloramphenicol ointment and 
the irregularity had fully resolved when the patient was re-examined 1 week later.

If the surgeon was concerned that the pupil was excessively dilated at the end of surgery, the pupil could be constricted by injecting Miochol (Bausch \& Lomb) into the anterior chamber. Pharmacy data was requested for a 12 month period before, and after, the introduction of Mydriasert. Prior to Mydriasert 191 doses of Miochol were ordered (1547 cataract operations performed) versus 200 doses following Mydriasert (1659 cataract operations performed). There was no significant difference in the rate of Miochol use before versus after the introduction of Mydriasert ( $p=0.83$, Fishers exact test) and hence the cost of Miochol was not considered in the economic model.

\section{Cost analysis of mydriasert compared with mydriatic eye drops}

The base case analysis showed that when Mydriasert substituted mydriatic eye drops, annual total costs decreased by $18 \%$, saving $£ 2111$ ( $£ 1.20$ per patient) (Table 2; Fig. 3). Annual total nurse time decreased from 235.1 hours to 44.1 hours when Mydriasert replaced mydriatic eye drops, which represented a total reduction in nursing costs from $£ 9873$ to $£ 1851$ (Table 2; Fig. 3). Sensitivity analysis confirmed that nurse time to instil mydriatic eye drops was a key cost driver in the economic model (data not shown).

A statistically significant reduction in iris hooks ordered was observed after the introduction of Mydriasert for cataract surgery. In the 8 months prior to Mydriasert use, 32 iris hooks were ordered $(2.78 \%, 1155$ cataract surgeries performed) compared to 12 iris hooks (1.0\%, 1198 cataract surgeries performed) in the subsequent 8 months following introduction of Mydriasert ( $\mathrm{p}=0.03$, chi-squared test).

Scenario analysis incorporating the incidence of use, surgeon time (10 minutes) and the cost of iris hooks ( $£ 54$ per case) into the economic model estimated a total annual cost saving of $£ 4559$ ( $£ 2.59$ per patient) using Mydriasert compared with mydriatic eye drops.

Table 2 Base case analysis for Mydriasert compared to eye drops (tropicamide [1\%] and phenylephrine [10\%]) to induce mydriasis for a patient cohort* undergoing cataract surgery from 2012-2013

\begin{tabular}{llll}
\hline & Mydriasert & Eye drops & Difference \\
\hline Mydriasert costs $(£)$ & 7598 & 0 & 7598 \\
Eye-drops costs $(£)$ & 0 & 1687 & -1687 \\
Nurse costs $(£)$ & 1851 & 9873 & -8022 \\
$\quad$ Annual total costs $(£)$ & 9449 & 11560 & -2111 \\
$\quad$ Costs per patient $(£)$ & 5.36 & 6.56 & -1.20 \\
Annual total nurse time (hours) & 44.1 & 235.1 & -191.0 \\
\hline
\end{tabular}

${ }^{*} \mathrm{~N}=1763$ cataract surgery patients and a single London hospital in one year. 2013 drug costs 2013 and 2012 nurse/consultant costs were used

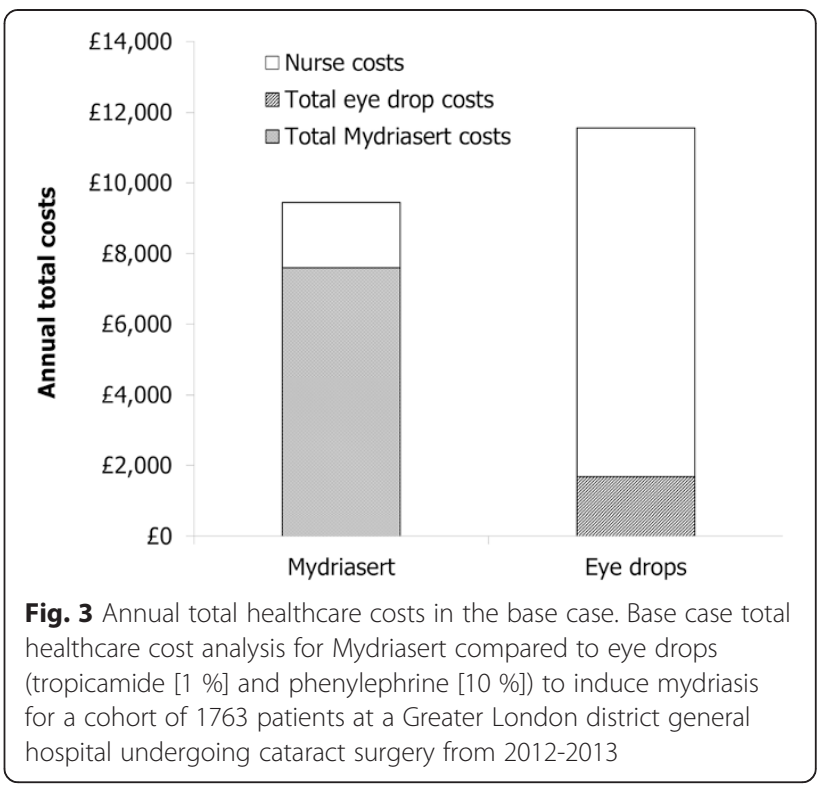

\section{Discussion}

A budget impact model was developed to estimate the drug, consumable and staff costs for achieving mydriasis using Mydriasert compared with mydriatic eye drops. The model was designed to assist NHS managers and local payers when considering the budget impact of introducing Mydriasert to their hospital settings. Interviews with clinicians and a clinical evaluation of cataract surgeries using Mydriasert at a district general hospital in Greater London provided input parameters for the model.

Using the designated hospital population of 1763 cataract surgery patients for the base case analysis, this study demonstrated that compared with traditional mydriatic eye drops, Mydriasert provided comparable clinical outcomes with overall savings in health-care costs mainly associated with reduced nurse time. This study also supports previous investigations which demonstrated the efficacy of Mydriasert to produce stable pupillary dilation [1-8]. After a single application of Mydriasert, sustained pupil dilation was achieved. The dilation achieved was suitable for the duration of cataract surgery enabling nursing staff to insert Mydriasert pellets for a group of patients simultaneously. This removed the need for repeated patient visits to increase efficiency, minimise other workload interruption, and provided the assurance that the pupil would stay dilated even if surgery was delayed. In contrast, the previous protocol used for pre-operative pupil dilatation at the district general hospital where the study was conducted required at least four instillations of mydriatic eye drops.

In practise, some ophthalmology units may use a less rigid dilation regime with regular monitoring, topping up mydriatic eye drops as necessary to ensure sufficient dilation is achieved prior to cataract surgery. However, if 
mydriatic eye drops are not re-administered in a timely manner the size of the pupil may be reduced and this is a recognised risk factor for the complication of posterior capsule rupture [13]. With the increasing age of the general population, the demand for cataract surgery is expected to increase in the future [14], thus cost-saving methods that improve the efficiency of healthcare practitioners should be considered.

In the scenario analysis, the number of iris hooks ordered reduced by $63 \%$ since the introduction of Mydriasert. This suggested that Mydriasert may reduce the frequency of iris hooks required during cataract surgery, subsequently reducing expenditure on hooks and the associated costs incurred by involving the surgeon's time. Further studies are required that actively exclude or control confounding variables around the measurement of iris hook usage.

Although the Mydriasert pellet is more expensive than mydriatic eye drops, the higher cost was offset by increased nurse time required for instillation of the eye drops. A limitation of the model was that a one-off cost of nurse training (which may be variable) was not included which could impact the cost savings achieved, however the model was designed to compare a steady state use of Mydriasert and mydriatic eye drops in clinical practice. Data collected during the first month of use report that the average time required for nurses to insert Mydriasert was 1.5 minutes; this time is likely to decrease as nurses gain more experience with Mydriasert. Since sensitivity analysis showed the nurse time was a key cost driver in the economic model (data not shown), the costs associated with Mydriasert could decrease further over time.

\section{Conclusions}

An economic analysis of a cohort of patients undergoing cataract surgery at a Greater London district general hospital showed that despite its higher unit cost, Mydriasert was cost saving overall compared with mydriatic eye drops. The reduction in costs was attributed to the decrease in nursing time required for Mydriasert compared with mydriatic eye drops thus; Mydriasert improved efficiency and could enable nurses to spend more time on other activities.

\section{Competing interests}

The current study was funded by Laboratoires Théa. AS, and SJ received consultation fees and travel/conference expenses for presenting the study from Laboratoires Théa. NL has no conflict of interest to declare.

\section{Acknowledgements}

Data from the current study has been presented as part of oral and poster presentations at the European Association for Vision and Eye Research congress, Nice France: $18^{\text {th }}-21^{\text {st }}$ September 2013 [11].

The authors acknowledge Sarah Collington, Ida Deleskog Lindstrom, and Richard Tolley at HERON ${ }^{\mathrm{TM}}$ Commercialization, PAREXEL International for their assistance with the preparation of the manuscript. The authors would also like to thank the healthcare professionals interviewed during model development.

\section{Author details}

1Department of Ophthalmology, Moorfields Eye Hospital NHS Foundation Trust, London, UK. ${ }^{2}$ Health Economic Modelling Unit, HERON ${ }^{\mathrm{TM}}$

Commercialization, PAREXEL International, London, UK. ${ }^{3}$ Department of Ophthalmology, Hillingdon Hospital NHS Foundation Trust, Uxbridge, UK.

Received: 18 December 2014 Accepted: 18 May 2015

Published online: 03 June 2015

\section{References}

1. Korobelnik JF, Tavera C, Renaud-Rougier MB, El MS, Colin J. The Mydriasert insert: an alternative to eye drops for preangiographic mydriasis. J Fr Ophtalmol. 2004;27:897-902.

2. Levet L, Touzeau O, Scheer S, Borderie V, Laroche L. A study of pupil dilation using the Mydriasert ophthalmic insert. J Fr Ophtalmol. 2004;27:1099-108.

3. Mouly S, Mahe I, Haouchine B, Sanson-le-Pors MJ, Blain P, Tillet Y, et al. Pharmacodynamics of a new ophthalmic mydriatic insert in healthy volunteers: potential alternative as drug delivery system prior to cataract surgery. Basic Clin Pharmacol Toxicol. 2006;98:547-54.

4. Morgado G, Barros P, Martins J, Lima A, Martins N. Comparative study of mydriasis in cataract surgery: topical versus Mydriasert versus intracameral mydriasis in cataract surgery. Eur J Ophthalmol. 2010;20:989-93.

5. Caruba T, Couffon-Partant C, Oliary J, Tadayoni R, Limelette N, Gaudric A. Efficacy and efficiency of preoperative mydriasis: drops versus ocular insert. J Fr Ophtalmol. 2006;29:789-95.

6. Torron C, Calvo P, Ruiz-Moreno O, Lecinena J, Perez-Inigo A: Use of a New Ocular Insert versus Conventional Mydriasis in Cataract Surgery. Biomed Res Int 2013, Published Online First: 30 May 2013. doi: 10.1155/2013/849349.

7. Nogueira H, Nogueira V, Silva S, Folgado A, Castanheira-Dinis A. Preoperative Mydriasis - Mydriasert Versus Ophthalmic Mydriatic Drop. Oftalmologia. 2009;33:165-70.

8. Saenz-de-Viteri M, Fernandez-Robredo P, de Nova E, Bonet-Farriol E, Sabater AL, Zarranz-Ventura J, et al. Comparative study measuring the dilatory effect of a mydriatic device (Mydriasert( $\left(^{\oplus}\right)$ ) versus topical drops. Int J Ophthalmol. 2013;6:801-4.

9. BNF. British National Formulary. http://www.bnfformularycomplete.com. 2013. 2013.

10. NICE. Medical Technologies Evaluation Programme - Methods guide. https://www.nice.org.uk/about/what-we-do/our-programmes/nice-guidance/nice-medical-technologies-guidance. 2011. 2012.

11. Shah A, Johal S, Lee N: Mydriasert pupillary dilation for cataract surgery: A clinical and economic study. Presented at 2013 European Association for Vision and Eye Research Conference. Acta Ophthalmologica. 2013;91:0.

12. PSSRU. Unit Costs of Health \& Social Care. http://www.pssru.ac.uk/projectpages/unit-costs/2012/ . 2012. 2013.

13. Narendran N, Jaycock P, Johnston RL, Taylor H, Adams M, Tole DM, et al. The Cataract National Dataset electronic multicentre audit of 55,567 operations: risk stratification for posterior capsule rupture and vitreous loss. Eye (Lond). 2009;23:31-7.

14. Kessel L. Can we meet the future demands for cataract surgery? Acta Ophthalmol. 2011;89:e289-90.

\section{Authors' contributions}

AS, SJ, and NL participated in the study design, conception, and development of the model protocol. SJ developed the economic model and performed the data analysis. All authors contributed to the manuscript development and read and approved the final manuscript. 